Pacific

Journal of

Mathematics

GENERALIZED FOCK SPACES AND WEYL COMMUTATION RELATIONS FOR THE DUNKL KERNEL

Fethi Soltani

Volume $214 \quad$ No. 2

April 2004 


\title{
GENERALIZED FOCK SPACES AND WEYL COMMUTATION RELATIONS FOR THE DUNKL KERNEL
}

\author{
Fethi Soltani
}

\begin{abstract}
In this paper we study a class of generalized Fock spaces associated with the Dunkl operators. Next we introduce the commutator relations between the Dunkl operators and multiplication operators which lead to a generalized class of Weyl commutation relations for the Dunkl kernel.
\end{abstract}

\section{Introduction.}

Fock space (called also Segal-Bargmann space [5]) is the Hilbert space of entire functions of $\mathbb{C}^{d}$ with inner product given by

$$
(f, g):=\frac{1}{\pi^{d}} \int_{\mathbb{C}^{d}} f(z) \overline{g(z)} e^{-|z|^{2}} d x d y, \quad z=x+i y,
$$

where

$$
|z|^{2}=\sum_{i=1}^{d} x_{i}^{2}+y_{i}^{2}, \quad d x d y=\prod_{i=1}^{d} d x_{i} d y_{i} .
$$

This space which associated with Fock's [12] realization of the creation and annihilation operators of Bose particles is studied by Bargmann [4]. Next, the ordinary Fock space $\mathcal{A}$ is the subject of many works $([\mathbf{5}, \mathbf{7}]$ and $[\mathbf{8}])$.

In 2001, M. Sifi and F. Soltani [21] introduced a Hilbert space $\mathcal{A}_{\gamma}$ of entire functions where the inner product is weighted by a generalized Gaussian distribution. On $\mathcal{A}_{\gamma}$ the Dunkl operator on the real line:

$$
T_{\gamma}(f)(z):=\frac{d}{d z} f(z)+\frac{2 \gamma}{z}\left[\frac{f(z)-f(-z)}{2}\right], \quad \gamma>0,
$$

and the multiplication by $z$ are adjoints and satisfy the commutation rule

$$
\left[T_{\gamma}, z\right]=I+2 \gamma B \text {, where } B f(x)=f(-x) .
$$

This commutator rule leads to a generalized class of Weyl commutation relations for the Dunkl kernel in the one dimensional.

In this paper we consider the differential-difference operators $T_{j}, j=$ $1, \ldots, d$, on $\mathbb{R}^{d}$ introduced by C.F. Dunkl in [9] and called Dunkl operators in the literature. These operators are very important in pure Mathematics and in Physics. They provide a useful tool in the study of special functions associated with root systems [10]. They are closely related to certain 
representations of degenerated affine Hecke algebras ([6] and [16]). Moreover the commutative algebra generated by these operators has been used in the study of certain exactly solvable models of quantum Mechanics, namely the Calogero-Sutherland-Moser models, which deal with systems of identical particles in the one dimensional space $([\mathbf{2}, \mathbf{3}]$ and $[\mathbf{1 4}])$.

The Dunkl kernel $E_{k}(x, y)$ is the unique solution of the initial problem

$$
T_{j}^{x} u(x, y)=y_{j} u(x, y) ; u(0, y)=1 ; j=1, \ldots, d,
$$

see $[\mathbf{1 0}, \mathbf{1 7}]$ and $[\mathbf{1 8}]$. This kernel has a unique holomorphic extension to $\mathbb{C}^{d} \times$ $\mathbb{C}^{d}$. Furthermore, the Dunkl kernel $E_{k}(z, w) ; z, w \in \mathbb{C}^{d}$ can be expanded in a power series in the form

$$
E_{k}(z, w)=\sum_{\nu \in \mathbb{N}^{d}} \varphi_{\nu}(z) \varphi_{\nu}(w)
$$

with some homogeneous orthonormal basis $\left\{\varphi_{\nu}\right\}_{\nu \in \mathbb{N}^{d}}$ of polynomials $([\mathbf{1 7}]$ and $[\mathbf{1 9}])$.

We introduce in this paper the generalized Fock space $\mathcal{A}_{k}$ associated with the Dunkl operators. This is a Hilbert space of functions $f$ on $\mathbb{C}^{d}$ which can be written $f(z)=\sum_{\nu \in \mathbb{N}^{d}} a_{\nu} \varphi_{\nu}(z)$ with

$$
\|f\|_{k}^{2}=(f, f)_{k}:=\sum_{\nu \in \mathbb{N}^{d}}\left|a_{\nu}\right|^{2}<\infty
$$

If $f, g \in \mathcal{A}_{k}$, having series expansions $f(z)=\sum_{\nu \in \mathbb{N}^{d}} a_{\nu} \varphi_{\nu}(z)$ and $g(z)=$ $\sum_{\nu \in \mathbb{N}^{d}} b_{\nu} \varphi_{\nu}(z)$. Then the inner product is given by the generalized spherical harmonics

$$
(f, g)_{k}=(f(T) \widetilde{g})(0),
$$

where $f(T)=f\left(T_{1}, \ldots, T_{d}\right)$ and $\widetilde{g}(z)=\sum_{\nu \in \mathbb{N}^{d}} \overline{b_{\nu}} \varphi_{\nu}(z)$.

The generalized Fock space $\mathcal{A}_{k}$, has also a reproducing kernel $\mathcal{K}$ given by $\mathcal{K}(z, w)=E_{k}(z, \bar{w}) ; z, w \in \mathbb{C}^{d}$. If $f \in \mathcal{A}_{k}$, then we have

$$
f(w)=\left(f, E_{k}(\cdot, \bar{w})\right)_{k}, \quad w \in \mathbb{C}^{d} .
$$

Thus the Dunkl kernel serves as the generalized Dirac delta function in $\mathcal{A}_{k}$.

The associated operators for the generalized Fock space $\mathcal{A}_{k}$ are $T_{j}$ and the multiplication operator by $z_{j}$. They are adjoints in $\mathcal{A}_{k}$ and satisfy a commutation rule:

$$
\left[T_{i}, z_{j}\right]=\delta_{i, j} I+\sum_{\alpha \in R_{+}} k(\alpha) \alpha_{i} \alpha_{j} B_{\alpha} ; \quad i, j=1, \ldots, d,
$$

where $B_{\alpha}$ a reflection operator, $k(\alpha)$ a multiplicity function and $R_{+}$is a positive root system.

These commutators rule lead to a generalized class of Weyl commutation relations for the Dunkl kernel.

These relations are studied in the classical case $(k=0)$ in $[\mathbf{1 3}]$. 
Throughout this paper we shall use the standard multi-index notations. This for multi-indices $\nu, s \in \mathbb{N}^{d}$, we write $|\nu|=\sum_{i=1}^{d} \nu_{i}, \nu !=\prod_{i=1}^{d} \nu_{i}$ !, $\left(\begin{array}{c}\nu \\ s\end{array}\right)=\prod_{i=1}^{d}\left(\begin{array}{c}\nu_{i} \\ s_{i}\end{array}\right)$ as well as $z^{\nu}=\prod_{i=1}^{d} z_{i}^{\nu_{i}}, D^{\nu}=\prod_{i=1}^{d} D_{i}^{\nu_{i}}$, for $z=$ $\left(z_{1}, \ldots, z_{d}\right) \in \mathbb{C}^{d}$ and any family $D=\left(D_{1}, \ldots, D_{d}\right)$ of commuting operators. Finally, we will need the partial ordering $\leq$ on $\mathbb{N}^{d}$, which is defined by $s \leq \nu \Leftrightarrow s_{i} \leq \nu_{i}, \quad i=1, \ldots, d$.

\section{Preliminaries.}

In this section we collect some notations and results on Dunkl operators and Dunkl kernel that will be important later on. General references here are $[\mathbf{9}, \mathbf{1 7}, \mathbf{1 8}, \mathbf{1 9}]$ and $[\mathbf{2 0}]$.

We consider $\mathbb{R}^{d}$ with the Euclidean scalar $\langle.,$.$\rangle and |x|=\sqrt{\langle x, x\rangle}$. On $\mathbb{C}^{d},|$.$| denotes also the standard Hermitian norm, while \langle z, w\rangle=\sum_{j=1}^{d} z_{j} w_{j}$ and $\ell(z)=\langle z, z\rangle$.

For $\alpha \in \mathbb{R}^{d} \backslash\{0\}$, let $\sigma_{\alpha}$ be the reflection in the hyperplane $H_{\alpha} \subset \mathbb{R}^{d}$ orthogonal to $\alpha$,

$$
\sigma_{\alpha} x:=x-\frac{2\langle\alpha, x\rangle}{|\alpha|^{2}} \alpha .
$$

A finite set $R \subset \mathbb{R}^{d} \backslash\{0\}$ is called a root system if $R \cap \mathbb{R} . \alpha=\{-\alpha, \alpha\}$ and $\sigma_{\alpha} R=R$ for all $\alpha \in R$. We assume that it is normalized by $|\alpha|^{2}=2$ for all $\alpha \in R$. For a given root system $R$ the reflections $\sigma_{\alpha}, \alpha \in R$ generated a finite group $G \subset O(d)$, the reflection group associated with $R$. All reflections in $G$ correspond to suitable pairs of roots. For a given $\beta \in H=\mathbb{R}^{d} \backslash \bigcup_{\alpha \in R} H_{\alpha}$, we fix the positive subsystem $R_{+}=\{\alpha \in R /\langle\alpha, \beta\rangle>0\}$, then for each $\alpha \in R$ either $\alpha \in R_{+}$or $-\alpha \in R_{+}$. The connected components of $H$ are called the Weyl chambers of $G$.

A function $k: R \rightarrow \mathbb{C}$ on a root system $R$ is called a multiplicity function if it is invariant under the action of the associated reflection group $G$. If one regards $k$ as a function on the corresponding reflections, this means that $k$ is constant on the conjugacy classes of reflections in $G$. For abbreviation, we introduce the index

$$
\gamma=\gamma(k):=\sum_{\alpha \in R_{+}} k(\alpha)
$$

Moreover, let $w_{k}$ denotes the weight function:

$$
w_{k}(x):=\prod_{\alpha \in R_{+}}|\langle\alpha, x\rangle|^{2 k(\alpha)}, \quad x \in \mathbb{R}^{d},
$$

which is $G$-invariant and homogeneous of degree $2 \gamma$. 
For $d=1$ and $G=\mathbb{Z}_{2}$, the multiplicity function $k$ is a simple parameter denoted $\gamma>0$ and

$$
w_{k}(x)=|x|^{2 \gamma}, \quad x \in \mathbb{R} .
$$

The Dunkl operators $T_{j} ; j=1, \ldots, d$, on $\mathbb{R}^{d}$ associated with the finite reflection group $G$ and multiplicity function $k$ are given for a function $f$ of class $C^{1}$ on $\mathbb{R}^{d}$, by

$$
T_{j} f(x):=\frac{\partial}{\partial x_{j}} f(x)+\sum_{\alpha \in R_{+}} k(\alpha) \alpha_{j} \frac{f(x)-f\left(\sigma_{\alpha} x\right)}{\langle\alpha, x\rangle} .
$$

In the case $k=0$, the $T_{j} ; j=1 \ldots, d$, reduce to the corresponding partial derivatives. In this paper we will assume throughout that $k \geq 0$.

For $y \in \mathbb{R}^{d}$, the initial problem

$$
\left\{\begin{array}{l}
T_{j}^{x} u(x, y)=y_{j} u(x, y) ; \quad j=1, \ldots, d, \\
u(0, y)=1
\end{array}\right.
$$

admits a unique analytic solution on $\mathbb{R}^{d}$, which will be denoted $E_{k}(x, y)$ and called the Dunkl kernel $([\mathbf{1 7}, \mathbf{1 8}, \mathbf{1 9}]$ and $[\mathbf{2 0}])$. This kernel has a unique holomorphic extension to $\mathbb{C}^{d} \times \mathbb{C}^{d}$.

\section{Examples.}

1) If $k=0$, then $E_{k}(z, w)=e^{\langle z, w\rangle}$ for $z, w \in \mathbb{C}^{d}$. (Recall that $\langle.,$.$\rangle was$ defined to be bilinear on $\mathbb{C}^{d} \times \mathbb{C}^{d}$.)

2) If $d=1$ and $G=\mathbb{Z}_{2}$, the Dunkl kernel is given by

$$
E_{\gamma}(z, w)=\Im_{\gamma-\frac{1}{2}}(z w)+\frac{z w}{2 \gamma+1} \Im_{\gamma+\frac{1}{2}}(z w),
$$

where

$$
\Im_{\gamma-\frac{1}{2}}(z w)=\Gamma\left(\gamma+\frac{1}{2}\right) \sum_{n=0}^{\infty} \frac{1}{n ! \Gamma\left(n+\gamma+\frac{1}{2}\right)}\left(\frac{z w}{2}\right)^{2 n},
$$

is the modified Bessel function of order $\gamma-\frac{1}{2}[\mathbf{2 1}]$.

Let $\mathcal{P}=\mathbb{C}\left[\mathbb{R}^{d}\right]$ denotes the $\mathbb{C}$ - Algebra of polynomial functions on $\mathbb{R}^{d}$ and $\mathcal{P}_{n}, n \in \mathbb{N}$, the subspace of homogeneous polynomials of degree $n$. In the context of generalized spherical harmonics, C.F. Dunkl in [9] introduced on $\mathcal{P}$ the following bilinear form:

$$
(p, q)_{k}:=(p(T) q)(0) ; \quad p, q \in \mathcal{P} .
$$

Here $p(T)$ is the operator derived from $p(x)$ by replacing $x_{i}$ by $T_{i}$. A useful collection of its properties can be found in [9] and [17]. We recall that $(., .)_{k}$ is symmetric, positive-definite and $(p, q)_{k}=0$, for $p \in \mathcal{P}_{n}, q \in \mathcal{P}_{m}$ with $n \neq m$. Moreover, for all $j=1, \ldots, d$ and $p, q \in \mathcal{P}$,

$$
\left(x_{j} p, q\right)_{k}=\left(p, T_{j} q\right)_{k} \text {. }
$$


Let $\left\{\varphi_{\nu}\right\}_{\nu \in \mathbb{N}^{d}}$ be an orthonormal basis of $\mathcal{P}$ with respect to the scalar product $(., .)_{k}$ such that $\varphi_{\nu} \in \mathcal{P}_{|\nu|}$ and the coefficients of the $\varphi_{\nu}$ are real. As $\mathcal{P}=\oplus_{n \in \mathbb{N}} \mathcal{P}_{n}$ and $\mathcal{P}_{n} \perp \mathcal{P}_{n}$ for $n \neq m$, the $\varphi_{\nu}$ with $|\nu|=n$ can for example be constructed by Gram-Schmidt orthogonalization within $\mathcal{P}_{n}$ from an arbitrary ordered real-coefficients basis of $\mathcal{P}_{n}$. If $k=0$ the canonical choice of the homogeneous orthonormal basis $\varphi_{\nu}$ is just $\varphi_{\nu}(x)=\frac{x^{\nu}}{\sqrt{\nu !}}$.

As in the classical case, M. Rösler obtained in [17, p. 524] the following connection of the basis $\varphi_{\nu}$ with the Dunkl kernel:

$$
E_{k}(z, w)=\sum_{\nu \in \mathbb{N}^{d}} \varphi_{\nu}(z) \varphi_{\nu}(w) ; \quad z, w \in \mathbb{C}^{d},
$$

where the convergence is normal on $\mathbb{C}^{d} \times \mathbb{C}^{d}$.

Example. If $d=1$ and $G=\mathbb{Z}_{2}$ every homogeneous orthonormal basis is of the form

$$
\varphi_{n}(z)=\frac{z^{n}}{\sqrt{b_{n}(\gamma)}}, \quad b_{n}(\gamma)=\frac{2^{n}([n / 2]) !}{\Gamma\left(\gamma+\frac{1}{2}\right)} \Gamma\left(\left[\frac{n+1}{2}\right]+\gamma+\frac{1}{2}\right) .
$$

Here $[n / 2]$ is the integer part of $n / 2$.

From (2), the Dunkl kernel $E_{k}$ possesses the following properties $([\mathbf{1 7}, \mathbf{1 9}]$ and [20]): For all $z, w \in \mathbb{C}^{d}$ and $\lambda \in \mathbb{C}$,

$$
\begin{gathered}
E_{k}(z, w)=E_{k}(w, z), \quad E_{k}(\lambda z, w)=E_{k}(z, \lambda w), \\
\overline{E_{k}(z, w)}=E_{k}(\bar{z}, \bar{w}), \quad E_{k}(z, \bar{z})=\sum_{\nu \in \mathbb{N}^{d}}\left|\varphi_{\nu}(z)\right|^{2}, \\
\left|E_{k}(z, w)\right| \leq e^{|z||w|} .
\end{gathered}
$$

In [18], M. Rösler establish the Bochner-type representation of the Dunkl kernel

$$
E_{k}(x, z)=\int_{\mathbb{R}^{d}} e^{\langle\xi, z\rangle} d \mu_{x}(\xi) ; \quad x \in \mathbb{R}^{d}, z \in \mathbb{C}^{d},
$$

where $\mu_{x}$ is a probability measure on $\mathbb{R}^{d}$ with support in $\left\{\xi \in \mathbb{R}^{d} /|\xi| \leq|x|\right\}$.

The Dunkl kernel $E_{k}$ is analytic on $\mathbb{C}^{d} \times \mathbb{C}^{d}$. Therefore, there exist unique analytic functions $m_{\nu}, \nu \in \mathbb{N}^{d}$, on $\mathbb{C}^{d}$ with

$$
E_{k}(z, w)=\sum_{\nu \in \mathbb{N}^{d}} \frac{m_{\nu}(z)}{\nu !} w^{\nu} ; \quad z, w \in \mathbb{C}^{d} .
$$

The restriction of $m_{\nu}$ to $\mathbb{R}^{d}$ are called the $\nu$-th moment functions $([\mathbf{1 8}, \mathbf{1 9}]$ and $[20])$. It is given explicitly by

$$
m_{\nu}(x)=\int_{\mathbb{R}^{d}} \xi^{\nu} d \mu_{x}(\xi), \quad x \in \mathbb{R}^{d},
$$

where $\mu_{x}$ is the measure given by (7). 
The functions $m_{\nu}$ are homogeneous polynomials of degree $|\nu|$. Among the applications of these moments, we mention the construction of martingales from Dunkl-type Markov processes [19].

\section{Fock spaces for the Dunkl kernel.}

In this section we define and study the generalized Fock space for the Dunkl kernel in $d$-dimensions.

Definition 1. The generalized Fock space $\mathcal{A}_{k}$ associated with the Dunkl operators is the space of holomorphic functions $f$ on $\mathbb{C}^{d}$ which can be written $f(z)=\sum_{\nu \in \mathbb{N}^{d}} a_{\nu} \varphi_{\nu}(z)$ with

$$
\|f\|_{k}^{2}:=\sum_{\nu \in \mathbb{N}^{d}}\left|a_{\nu}\right|^{2}<\infty .
$$

Hence the inner product in $\mathcal{A}_{k}$ is given for $f(z)=\sum_{\nu \in \mathbb{N}^{d}} a_{\nu} \varphi_{\nu}(z) \in \mathcal{A}_{k}$ and $g(z)=\sum_{\nu \in \mathbb{N}^{d}} b_{\nu} \varphi_{\nu}(z) \in \mathcal{A}_{k}$, by

$$
(f, g)_{k}:=\sum_{\nu \in \mathbb{N}^{d}} a_{\nu} \overline{b_{\nu}}
$$

Remark. If $k=0, \mathcal{A}_{0}$ is the ordinary Fock space $\mathcal{A}[4]$.

\section{Proposition 1.}

i) If $f, g \in \mathcal{A}_{k}$ with $f(z)=\sum_{\nu \in \mathbb{N}^{d}} a_{\nu} \varphi_{\nu}(z)$ and $g(z)=\sum_{\nu \in \mathbb{N}^{d}} b_{\nu} \varphi_{\nu}(z)$, we have

$$
(f, g)_{k}=(f(T) \widetilde{g})(0),
$$

where $\widetilde{g}(z)=\sum_{\nu \in \mathbb{N}^{d}} \overline{b_{\nu}} \varphi_{\nu}(z)$.

ii) If $f \in \mathcal{A}_{k}$ with $f(z)=\sum_{\nu \in \mathbb{N}^{d}} a_{\nu} \varphi_{\nu}(z)$, we have

$$
|f(z)| \leq e^{|z|^{2} / 2}\|f\|_{k}
$$

Proof. i) From $[\mathbf{1 7}$, p. 529], we have

$$
\left(\varphi_{\nu}(T) \varphi_{s}\right)(0)=\delta_{\nu, s}
$$

where $\delta_{\nu, s}$ is the Kronecker symbol.

Thus

$$
(f, g)_{k}=\sum_{\nu, s \in \mathbb{N}^{d}} a_{\nu} \overline{b_{s}}\left(\varphi_{\nu}(T) \varphi_{s}\right)(0) .
$$

Using the continuously of the inner product, we obtain the result.

ii) Using Cauchy-Schwarz's inequality, then

$$
|f(z)|^{2} \leq\left[\sum_{\nu \in \mathbb{N}^{d}}\left|a_{\nu}\right|^{2}\right]\left[\sum_{\nu \in \mathbb{N}^{d}}\left|\varphi_{\nu}(z)\right|^{2}\right]=\|f\|_{k}^{2} E_{k}(z, \bar{z}) .
$$


Thus

$$
|f(z)| \leq\left[E_{k}(z, \bar{z})\right]^{1 / 2}\|f\|_{k} .
$$

The result follows from the inequality (6).

From Proposition 1 ii), the map $f \rightarrow f(z), z \in \mathbb{C}^{d}$, is a continuous linear functional on $\mathcal{A}_{k}$. Thus from Riesz theorem $[\mathbf{1}], \mathcal{A}_{k}$ has a reproducing kernel.

Proposition 2. The function $\mathcal{K}$ given for $w, z \in \mathbb{C}^{d}$, by

$$
\mathcal{K}(z, w)=E_{k}(z, \bar{w}),
$$

is a reproducing kernel for the generalized Fock spaces $\mathcal{A}_{k}$, that is:

i) For every $w \in \mathbb{C}^{d}$, the function $z \rightarrow \mathcal{K}(z, w)$ belongs to $\mathcal{A}_{k}$.

ii) The reproducing property: For every $w \in \mathbb{C}^{d}$ and $f \in \mathcal{A}_{k}$, we have

$$
(f, \mathcal{K}(\cdot, w))_{k}=f(w) .
$$

Proof. i) Using (5) and (6), we deduce for $w \in \mathbb{C}^{d}$,

$$
\left\|E_{k}(\cdot, \bar{w})\right\|_{k}^{2}=\sum_{\nu \in \mathbb{N}^{d}}\left|\varphi_{\nu}(\bar{w})\right|^{2}=E_{k}(w, \bar{w}) \leq e^{|w|^{2}},
$$

which proves i).

ii) If $f(z)=\sum_{\nu \in \mathbb{N}^{d}} a_{\nu} \varphi_{\nu}(z) \in \mathcal{A}_{k}$, it follows from (9) that

$$
\left(f, E_{k}(\cdot, \bar{w})\right)_{k}=\sum_{\nu \in \mathbb{N}^{d}} a_{\nu} \overline{\varphi_{\nu}(\bar{w})}=f(w) .
$$

\section{Corollary 1.}

i) The set $\left\{E_{k}(\cdot, \bar{w}), w \in \mathbb{C}^{d}\right\}$ is complete in $\mathcal{A}_{k}$.

ii) For all $z, w \in \mathbb{C}^{d}$, we have

$$
E_{k}(\bar{z}, w)=\left(E_{k}(\cdot, \bar{z}), E_{k}(\cdot, \bar{w})\right)_{k} .
$$

iii) Let $m \in \mathbb{N} \backslash\{0\}$ and $z_{1}, z_{2}, \ldots, z_{m} \in \mathbb{C}^{d}$, with $z_{i} \neq z_{j}$, then

$$
\operatorname{det}\left[E_{k}\left(\bar{z}_{i}, z_{j}\right)\right]_{i, j=1}^{m}>0 .
$$

Notation. We denote by $L^{2}\left(\mu_{k}\right)$ the Hilbert space of measurable functions on $\mathbb{R}^{d}$, for which

$$
\|f\|_{2, k}:=\left[\int_{\mathbb{R}^{d}}|f(x)|^{2} d \mu_{k}(x)\right]^{1 / 2}<\infty .
$$

Here $\mu_{k}$ is the measure defined on $\mathbb{R}^{d}$, by

$$
d \mu_{k}(x):=c_{k} w_{k}(x) d x, \text { with } c_{k}=\left(\int_{\mathbb{R}^{d}} e^{-|x|^{2}} d \mu_{k}(x)\right)^{-1},
$$

is the Mehta-type constant. 
In the next part of this section we establish the unitary equivalence of $L^{2}\left(\mu_{k}\right)$ and $\mathcal{A}_{k}$. First we recall some properties of the generalized Hermite functions $([\mathbf{1 7}]$ and $[\mathbf{1 9}])$ :

Definition 2. The generalized Hermite polynomials $\left\{H_{\nu}\right\}_{\nu \in \mathbb{N}^{d}}$ associated with the basis $\left\{\varphi_{\nu}\right\}_{\nu \in \mathbb{N}^{d}}$ on $\mathbb{C}^{d}$, are given by

$$
H_{\nu}(z):=2^{|\nu|} e^{-\triangle_{k} / 4} \varphi_{\nu}(z)=2^{|\nu|} \sum_{n=0}^{[|\nu| / 2]} \frac{(-1)^{n}}{2^{2 n} n !} \triangle_{k}^{n} \varphi_{\nu}(z),
$$

where $\triangle_{k}=\sum_{i=1}^{d} T_{i}^{2}$ is the Dunkl Laplacian [17].

Moreover, we define the generalized Hermite functions on $\mathbb{C}^{d}$, by

$$
h_{\nu}(z):=2^{-|\nu| / 2} e^{-\ell(z) / 2} H_{\nu}(z) .
$$

\section{Examples.}

1) If $k=0$, we obtain

$$
H_{\nu}(x)=\frac{2^{|\nu|}}{\sqrt{\nu !}} \prod_{i=1}^{d} e^{-\frac{1}{4} \frac{\partial^{2}}{\partial x_{i}{ }^{2}}}\left(x_{i}^{\nu_{i}}\right)=\frac{1}{\sqrt{\nu !}} \prod_{i=1}^{d} \widehat{H}_{\nu_{i}}\left(x_{i}\right), \quad x \in \mathbb{R}^{d},
$$

where

$$
\widehat{H}_{x_{i}}=(-1)^{\nu_{i}} e^{x_{i}^{2}} \frac{\partial^{\nu_{i}}}{\partial x_{i}^{\nu_{i}}}\left(e^{-x_{i}^{2}}\right) .
$$

2) If $d=1$ and $G=\mathbb{Z}_{2}$, we obtain

$$
H_{n}(z)=\sum_{i=0}^{[n / 2]} \frac{(-1)^{i}}{i ! b_{n-2 i}(\gamma)}(2 x)^{n-2 i}, \quad x \in \mathbb{R},
$$

where $b_{n}(\gamma)$ are the constants given by (3).

The following lemma is shown in [17, p. 525-529]:

\section{Lemma 1.}

i) The set $\left\{h_{\nu}\right\}_{\nu \in \mathbb{N}^{d}}$ is an orthonormal basis of $L^{2}\left(\mu_{k}\right)$.

ii) For all $z, w \in \mathbb{C}^{d}$, there is a generating function for the generalized Hermite polynomials,

$$
e^{-\ell(w)} E_{k}(2 z, w)=\sum_{\nu \in \mathbb{N}^{d}} h_{\nu}(z) \varphi_{\nu}(w) .
$$

Notation. We denote by $U_{k}$ the kernel given for $z, w \in \mathbb{C}^{d}$, by

$$
U_{k}(z, w):=e^{-(\ell(z)+\ell(w)) / 2} E_{k}(\sqrt{2} z, w) .
$$

Lemma 2. For $w, z \in \mathbb{C}^{d}$, we have

$$
U_{k}(z, w)=\sum_{\nu \in \mathbb{N}^{d}} h_{\nu}(z) \varphi_{\nu}(w) .
$$


Proof. From Definition 2, we have

$$
\sum_{\nu \in \mathbb{N}^{d}} h_{\nu}(z) \varphi_{\nu}(w)=e^{-\ell(z) / 2} \sum_{\nu \in \mathbb{N}^{d}} 2^{-|\nu| / 2} H_{\nu}(z) \varphi_{\nu}(w) .
$$

As $\varphi_{\nu}$ is homogeneous of degree $|\nu|$, then

$$
\varphi_{\nu}\left(\frac{w}{\sqrt{2}}\right)=2^{-|\nu| / 2} \varphi_{\nu}(w)
$$

Thus

$$
\sum_{\nu \in \mathbb{N}^{d}} h_{\nu}(z) \varphi_{\nu}(w)=e^{-\ell(z) / 2} \sum_{\nu \in \mathbb{N}^{d}} H_{\nu}(z) \varphi_{\nu}\left(\frac{w}{\sqrt{2}}\right) .
$$

Applying Lemma 1 ii) and (4), we obtain

$$
\sum_{\nu \in \mathbb{N}^{d}} h_{\nu}(z) \varphi_{\nu}(w)=e^{-(\ell(z)+\ell(w)) / 2} E_{k}\left(2 z, \frac{w}{\sqrt{2}}\right)=U_{k}(z, w) .
$$

\section{Lemma 3.}

i) For all $z, w \in \mathbb{C}^{d}$, we have

$$
E_{k}(z, w)=\int_{\mathbb{R}^{d}} U_{k}(z, x) U_{k}(w, x) d \mu_{k}(x) .
$$

ii) For all $z \in \mathbb{C}^{d}$, the function $x \rightarrow U_{k}(z, x)$ belongs to $L^{2}\left(\mu_{k}\right)$, and we have

$$
\left\|U_{k}(z, \cdot)\right\|_{2, k}^{2}=E_{k}(z, \bar{z}) .
$$

iii) For all $x \in \mathbb{R}^{d}$, the function $z \rightarrow U_{k}(z, x)$ belongs to $\mathcal{A}_{k}$, and we have

$$
\left\|U_{k}(\cdot, x)\right\|_{k}^{2}=e^{-3|x|^{2}} E_{k}(2 x, x) .
$$

Proof. i) We put

$$
I=\int_{\mathbb{R}^{d}} U_{k}(z, x) U_{k}(w, x) d \mu_{k}(x) .
$$

From (10), we have

$$
I=e^{-(\ell(z)+\ell(w)) / 2} \int_{\mathbb{R}^{d}} e^{-|x|^{2}} E_{k}(\sqrt{2} z, x) E_{k}(\sqrt{2} w, x) d \mu_{k}(x) .
$$

So from $[\mathbf{1 7}$, p. 523] and (4), we get

$$
\int_{\mathbb{R}^{d}} e^{-|x|^{2}} E_{k}(\sqrt{2} z, x) E_{k}(\sqrt{2} w, x) d \mu_{k}(x)=e^{(\ell(z)+\ell(w)) / 2} E_{k}(z, w),
$$

which proves i).

ii) This assertion follows from i) and (5).

iii) For $z \in \mathbb{C}^{d}$, we put

$$
\phi(z):=e^{-(\ell(z)+\ell(\bar{z})) / 2} .
$$


Let $x \in \mathbb{R}^{d}$, then from Proposition 2 ii), (10) and (4), we have

$$
\left\|U_{k}(\cdot, x)\right\|_{k}^{2}=e^{-|x|^{2}}\left(\phi(\cdot) E_{k}(\cdot, \sqrt{2} x), E_{k}(\cdot, \sqrt{2} x)\right)_{k}=e^{-3|x|^{2}} E_{k}(2 x, x) .
$$

Definition 3. The chaotic transform $\mathcal{C}_{k}$ (also called $S$-transform in the stochastic calculus [15]) is the transformation defined on $L^{2}\left(\mu_{k}\right)$, by

$$
\mathcal{C}_{k}(f)(z):=\int_{\mathbb{R}^{d}} U_{k}(z, x) f(x) d \mu_{k}(x), \quad z \in \mathbb{C}^{d} .
$$

Remark. The basis elements of $L^{2}\left(\mu_{k}\right)$ and $\mathcal{A}_{k}$ are called chaos. In the following theorem we shall prove that the transformation $\mathcal{C}_{k}$ maps the chaos of $L^{2}\left(\mu_{k}\right)$ to these of $\mathcal{A}_{k}$.

Theorem 1. The chaotic transform $\mathcal{C}_{k}$ is a unitary mapping of $L^{2}\left(\mu_{k}\right)$ on $\mathcal{A}_{k}$. Moreover, the basis elements are related by

$$
\mathcal{C}_{k}\left(h_{\nu}\right)=\varphi_{\nu}
$$

Proof. It follows directly from Lemma 1 i) and Lemma 2, that for $\nu \in \mathbb{N}^{d}$,

$$
\mathcal{C}\left(h_{\nu}\right)(z)=\int_{\mathbb{R}^{d}} U_{k}(z, x) h_{\nu}(x) d \mu_{k}(x)=\varphi_{\nu}(z), \quad z \in \mathbb{C}^{d} .
$$

Consequently $\mathcal{C}_{k}$ maps the subspace generated by the family $\left\{h_{\nu}\right\}_{\nu \in \mathbb{N}^{d}}$ into the polynomials in $\mathcal{A}_{k}$. Thus $\mathcal{C}_{k}$ maps a dense set in $L^{2}\left(\mu_{k}\right)$ into a dense set in $\mathcal{A}_{k}$. Further, if $f \in L^{2}\left(\mu_{k}\right)$, then $f(z)=\sum_{\nu \in \mathbb{N}^{d}} a_{\nu} h_{\nu}(x)$. For $\nu \in \mathbb{N}^{d}$, let $f_{N}(x)=\sum_{j=0}^{N} \sum_{|\nu|=j} a_{\nu} h_{\nu}(x), x \in \mathbb{R}$. Then

$$
\mathcal{C}_{k}\left(f_{N}\right)(z)=\sum_{j=0}^{N} \sum_{|\nu|=j} a_{\nu} \varphi_{\nu}(z) ; \lim _{N \rightarrow \infty}\left\|f-f_{N}\right\|_{2, k}=0 .
$$

On the other hand, from Hölder's inequality and Lemma 3 ii), we have

$$
\left|\mathcal{C}_{k}\left(f-f_{N}\right)(z)\right| \leq\left[E_{k}(z, \bar{z})\right]^{1 / 2}\left\|f-f_{N}\right\|_{2, k} .
$$

Thus we obtain

$$
\mathcal{C}_{k}(f)(z)=\sum_{\nu \in \mathbb{N}^{d}} a_{\nu} \varphi_{\nu}(z)
$$

Hence

$$
\left\|\mathcal{C}_{k}(f)\right\|_{k}^{2}=\sum_{\nu \in \mathbb{N}^{d}}\left|a_{\nu}\right|^{2}=\|f\|_{2, k}^{2} .
$$

It follows that $\mathcal{C}_{k}$ is a unitary transformation from $L^{2}\left(\mu_{k}\right)$ into $\mathcal{A}_{k}$.

Clearly, if $g(z)=\sum_{\nu \in \mathbb{N}^{d}} a_{\nu} \varphi_{\nu}(z) \in \mathcal{A}_{k}$, we have

$$
\mathcal{C}_{k}^{-1}(g)(x)=\sum_{\nu \in \mathbb{N}^{d}} a_{\nu} h_{\nu}(x), \quad x \in \mathbb{R}^{d} .
$$

Which completes the proof. 
Proposition 3. If $g \in \mathcal{A}_{k}$, we have

$$
\mathcal{C}_{k}^{-1}(g)(x)=\left(g, U_{k}(\cdot, x)\right)_{k}, \quad x \in \mathbb{R}^{d} .
$$

Proof. Let $g \in \mathcal{A}_{k}$. We put for $x \in \mathbb{R}^{d}$,

$$
\Psi_{k}(g)(x)=\left(g, U_{k}(\cdot, x)\right)_{k} .
$$

Using Lemma 2, Lemma 3 iii) and the same method as in the proof of Theorem 1 we obtain

$$
\Psi_{k}(g)(x)=\sum_{\nu \in \mathbb{N}^{d}} a_{\nu} h_{\nu}(x)=\mathcal{C}_{k}^{-1}(g)(x), \quad x \in \mathbb{R}^{d} .
$$

\section{Commutators and Weyl relations for the Dunkl kernel.}

We define the multiplication operators $Q_{i} ; i=1, \ldots, d$ on $\mathcal{A}_{k}$ by

$$
Q_{i} f(z):=z_{i} f(z), \quad z \in \mathbb{C}^{d} .
$$

We denote also by $T_{i} ; i=1, \ldots, d$ the operators defined on $\mathcal{A}_{k}$.

Let

$$
\begin{aligned}
\mathcal{D}\left(Q_{i}\right) & =\left\{f \in \mathcal{A}_{k} / Q_{i}(f) \in \mathcal{A}_{k}\right\}, \\
\mathcal{D}\left(T_{i}\right) & =\left\{f \in \mathcal{A}_{k} / T_{i} f \in \mathcal{A}_{k}\right\}
\end{aligned}
$$

denote the domains of $Q_{i}$ and $T_{i}$ respectively.

We denote by [.,.] the commutator product $([A, B]=A B-B A)$. As in $[\mathbf{1 1}]$, we have the following relations:

Lemma 4. The operators $Q_{i}$ and $T_{i}, i=1, \ldots, d$ satisfy on $\mathcal{A}_{k}$ the commutation relations:

$$
\begin{aligned}
& {\left[T_{i}, T_{j}\right]=\left[Q_{i}, Q_{j}\right]=0 ; \quad i, j=1, \ldots, d,} \\
& {\left[T_{i}, Q_{j}\right]=\delta_{i, j} I+\sum_{\alpha \in R_{+}} k(\alpha) \alpha_{i} \alpha_{j} B_{\alpha} ; \quad i, j=1, \ldots, d,}
\end{aligned}
$$

where $I$ the identity operator and $B_{\alpha}$ is the reflection operator $\left(B_{\alpha}^{2}=I\right)$ given by

$$
B_{\alpha} f(z)=f\left(\sigma_{\alpha} z\right) .
$$

Proof. Using the fact that $\sigma_{\alpha}^{2}=i d$ and $\left\langle\alpha, \sigma_{\alpha} z\right\rangle=-\langle\alpha, z\rangle$, we obtain

$$
T_{i} T_{j} f(z)=T_{i}\left(\frac{\partial}{\partial z_{j}} f\right)(z)+\sum_{\alpha \in R_{+}} k(\alpha) \alpha_{j} \frac{\partial}{\partial z_{i}}\left(\frac{f(z)-f\left(\sigma_{\alpha} z\right)}{\langle\alpha, z\rangle}\right) .
$$

Since

$$
\frac{\partial}{\partial z_{i}}\left(f\left(\sigma_{\alpha} z\right)\right)=\frac{\partial}{\partial z_{i}} f\left(\sigma_{\alpha} z\right)-\sum_{\ell=1}^{d} \alpha_{i} \alpha_{\ell} \frac{\partial}{\partial z_{\ell}} f\left(\sigma_{\alpha} z\right)
$$


we have

$$
\begin{aligned}
T_{i} T_{j} f(z)= & -\frac{\partial^{2}}{\partial z_{j} \partial z_{i}} f(z)+T_{i}\left(\frac{\partial}{\partial z_{j}} f\right)(z)+T_{j}\left(\frac{\partial}{\partial z_{i}} f\right)(z) \\
& -\sum_{\alpha \in R_{+}} k(\alpha) \alpha_{i} \alpha_{j}\left[\frac{f(z)-f\left(\sigma_{\alpha} z\right)}{(\langle\alpha, z\rangle)^{2}}-\sum_{\ell=1}^{d} \alpha_{\ell} \frac{\frac{\partial}{\partial z_{\ell}} f\left(\sigma_{\alpha} z\right)}{\langle\alpha, z\rangle}\right] .
\end{aligned}
$$

Thus

$$
\left[T_{i}, T_{j}\right] f(z)=0 .
$$

The other relations are evident.

\section{Proposition 4. Let}

$$
\begin{aligned}
f(z) & =\sum_{\nu \in \mathbb{N}^{d}} a_{\nu} \varphi_{\nu}(z) \in \mathcal{D}\left(Q_{i}\right) \quad \text { and } \\
g(z) & =\sum_{\nu \in \mathbb{N}^{d}} a_{\nu} \varphi_{\nu}(z) \in \mathcal{D}\left(T_{i}\right), \quad \text { then } \\
\left(Q_{i} f, g\right)_{k} & =\left(f, T_{i} g\right)_{k} .
\end{aligned}
$$

Proof. Applying Proposition 1 i), we get

$$
\left(Q_{i} f, g\right)_{k}=\left(Q_{i} f(T) \widetilde{g}\right)(0)=\sum_{\nu, s \in \mathbb{N}^{d}} a_{\nu} \overline{b_{s}} T_{i} \varphi_{\nu}(T) \varphi_{s}(0) .
$$

Then from (12) we obtain

$$
\left(Q_{i} f, g\right)_{k}=\sum_{\nu, s \in \mathbb{N}^{d}} a_{\nu} \overline{b_{s}} \varphi_{\nu}(T) T_{i} \varphi_{s}(0)=\left(f, T_{i} g\right)_{k} .
$$

Lemma 5. If $f \in \mathcal{A}_{k}$, then $B_{\alpha} f \in \mathcal{A}_{k}$, and we have

$$
\left\|Q_{i} f\right\|_{k}^{2}=\left\|T_{i} f\right\|_{k}^{2}+\|f\|_{k}^{2}+\sum_{\alpha \in R_{+}} k(\alpha) \alpha_{i}^{2}\left(f, B_{\alpha} f\right)_{k},
$$

where $B_{\alpha}$ is the operator given by (14).

Proof. Let $f \in \mathcal{A}_{k}$. Applying the chaotic transform, in view of Theorem 1, it suffices to show that $\mathcal{C}_{k}^{-1}\left(B_{\alpha} f\right) \in L^{2}\left(\mu_{k}\right)$. From (11), we have

$$
\mathcal{C}_{k}^{-1}\left(B_{\alpha} f\right)(x)=\mathcal{C}_{k}^{-1}(f)\left(\sigma_{\alpha} x\right), \quad x \in \mathbb{R}^{d} .
$$

Putting $u=\sigma_{\alpha} x$, we get

$$
d \mu_{k}(x)=\left|J_{\alpha}\right| d \mu_{k}(u) \quad \text { where } \quad J_{\alpha}=\operatorname{det}\left[\delta_{i, j}-\alpha_{i} \alpha_{j}\right]_{i, j=1}^{d} .
$$

Since $J_{\alpha}=-1$, we obtain

$$
\left\|\mathcal{C}_{k}^{-1}\left(B_{\alpha} f\right)\right\|_{2, k}^{2}=\int_{\mathbb{R}^{d}}\left|\mathcal{C}_{k}^{-1}(f)(u)\right|^{2} d \mu_{k}(u) .
$$


Which proves that $B_{\alpha} f \in \mathcal{A}_{k}$.

On the other hand, from Proposition 4, we deduce

$$
\left\|Q_{i} f\right\|_{k}^{2}=\left(f, T_{i} Q_{i} f\right)_{k} .
$$

But from (13), we have

$$
T_{i} Q_{i} f=Q_{i} T_{i} f+f+\sum_{\alpha \in R_{+}} k(\alpha) \alpha_{i}^{2} B_{\alpha} f
$$

Thus

$$
\left\|Q_{i} f\right\|_{k}^{2}=\left(f, Q_{i} T_{i} f\right)_{k}+\|f\|_{k}^{2}+\sum_{\alpha \in R_{+}} k(\alpha) \alpha_{i}^{2}\left(f, B_{\alpha} f\right)_{k} .
$$

Using another time Proposition 4, we obtain the result.

Proposition 5. The operators $Q_{i}$ and $T_{i}$ are closed densely defined operators on $\mathcal{A}_{k}$, and we have

$$
\mathcal{D}\left(Q_{i}\right)=\mathcal{D}\left(T_{i}\right) ; \quad Q_{i}^{*}=T_{i} ; \quad T_{i}^{*}=Q_{i},
$$

where $Q_{i}^{*}$ and $T_{i}^{*}$ are the adjoints operators of $Q_{i}$ and $T_{i}$, respectively.

Proof. These results follow from [4, Theorem 1.2], Lemma 5 and Proposition 4 by using the same method as [21, Proposition 6].

Lemma 6. For $\nu \in \mathbb{N}^{d} \backslash\{0\}$, we have the following relations:

i)

$$
\begin{aligned}
{\left[T^{\nu}, Q_{j}\right]=} & \nu_{j} T_{1}^{\nu_{1}} \ldots T_{i-1}^{\nu_{i-1}} T_{i}^{\nu_{i}-1} T_{i+1}^{\nu_{i+1}} \ldots T_{d}^{\nu_{d}} \\
& +B_{\alpha} \sum_{i=1}^{d} \sum_{\ell=0}^{\nu_{i}-1} \sum_{\alpha \in R_{+}} k(\alpha) \alpha_{i} \alpha_{j} H_{1}^{\nu_{1}} \ldots H_{i-1}^{\nu_{i-1}} H_{i}^{\ell} T_{i}^{\nu_{i}-\ell-1} T_{i+1}^{\nu_{i+1}} \ldots T_{d}^{\nu_{d}},
\end{aligned}
$$

where $H_{i} ; i=1, \ldots, d$, are given by the differential-difference operators

$$
H_{i}=-T_{i}+2 \frac{\partial}{\partial x_{i}}-\sum_{\ell=1}^{d} \alpha_{i} \alpha_{\ell} \frac{\partial}{\partial x_{\ell}} .
$$

ii)

$$
\begin{aligned}
{\left[T_{j}, Q^{\nu}\right]=} & \nu_{j} Q_{1}^{\nu_{1}} \ldots Q_{i-1}^{\nu_{i-1}} Q_{i}^{\nu_{i}-1} Q_{i+1}^{\nu_{i+1}} \ldots Q_{d}^{\nu_{d}} \\
& +B_{\alpha} \sum_{i=1}^{d} \sum_{\ell=0}^{\nu_{i}-1} \sum_{\alpha \in R_{+}} k(\alpha) \alpha_{i} \alpha_{j} Z_{1}^{\nu_{1}} \ldots Z_{i-1}^{\nu_{i-1}} Z_{i}^{\ell} Q_{i}^{\nu_{i}-\ell-1} Q_{i+1}^{\nu_{i+1}} \ldots Q_{d}^{\nu_{d}},
\end{aligned}
$$

where $Z_{i} ; i=1, \ldots, d$, are the multiplication operators given by

$$
Z_{i}=Q_{i}-\sum_{\ell=1}^{d} \alpha_{i} \alpha_{\ell} Q_{\ell}
$$


Proof. From (13), we have

$$
\begin{aligned}
{\left[T_{i}^{\nu_{i}}, Q_{j}\right] } & =\sum_{\ell=0}^{\nu_{i}-1} T_{i}^{\ell}\left[T_{i}, Q_{j}\right] T_{i}^{\nu_{i}-\ell-1} \\
& =\nu_{i} \delta_{i, j} T_{i}^{\nu_{i}-1}+\sum_{\ell=0}^{\nu_{i}-1} k(\alpha) \alpha_{i} \alpha_{j} T_{i}^{\ell} B_{\alpha} T_{i}^{\nu_{i}-\ell-1} .
\end{aligned}
$$

From this equality, we get

$$
\begin{aligned}
{\left[T^{\nu}, Q_{j}\right]=} & \sum_{i=1}^{d} T_{1}^{\nu_{1}} \ldots T_{i-1}^{\nu_{i-1}}\left[T_{i}^{\nu_{i}}, Q_{j}\right] T_{i+1}^{\nu_{i+1}} \ldots T_{d}^{\nu_{d}} \\
= & \nu_{j} T_{1}^{\nu_{1}} \ldots T_{i-1}^{\nu_{i-1}} T_{i}^{\nu_{i}-1} T_{i+1}^{\nu_{i+1}} \ldots T_{d}^{\nu_{d}} \\
& +\sum_{i=1}^{d} \sum_{\ell=0}^{\nu_{i}-1} \sum_{\alpha \in R_{+}} k(\alpha) \alpha_{i} \alpha_{j} T_{1}^{\nu_{1}} \ldots T_{i-1}^{\nu_{i-1}} T_{i}^{\ell} B_{\alpha} T_{i}^{\nu_{i}-\ell-1} T_{i+1}^{\nu_{i+1}} \ldots T_{d}^{\nu_{d}} .
\end{aligned}
$$

But

where

$$
T_{i}^{\nu_{i}} B_{\alpha}=B_{\alpha} H_{i}^{\nu_{i}}
$$

$$
H_{i}=-T_{i}+2 \frac{\partial}{\partial x_{i}}-\sum_{\ell=1}^{d} \alpha_{i} \alpha_{\ell} \frac{\partial}{\partial x_{\ell}} .
$$

Thus we obtain Assertion i). And similarly we get ii).

Notation. For $x \in \mathbb{R}^{d}$ and $z \in \mathbb{C}^{d}$, we denote by

$$
I_{k}(z, x):=\frac{E_{k}(z, x)-E_{k}\left(z, \sigma_{\alpha} x\right)}{\langle\alpha, x\rangle} .
$$

From $\left[\mathbf{1 7}\right.$, p. 533], we can write the function $I_{k}(z, x)$ in the form

$$
I_{k}(z, x)=\left\langle\nabla_{x} E_{k}(z, x), \alpha\right\rangle+\frac{1}{2}\langle\alpha, x\rangle \alpha^{t} D_{x}^{2} E_{k}(z, \xi) \alpha,
$$

with some $\xi$ on the line segment between $x$ and $\sigma_{\alpha} x$.

(Here $\nabla$ and $D^{2} f(x)$ denote the usual gradient and Hessian of $f$ in $x$.)

Lemma 7. For $a, b \in \mathbb{C}^{d}$, we have the following commutation relations:

i) $\left[E_{k}(a, T), Q_{j}\right]=a_{j} E_{k}(a, T)-R_{k, j}(a, T)$, where

$$
\begin{aligned}
& R_{k, j}(a, T)= \\
& \sum_{\alpha \in R_{+}} k(\alpha) \alpha_{j} I_{k}(a, T) \\
& -B_{\alpha} \sum_{\substack{\nu \in \mathbb{N}^{d} \\
\alpha \in R_{+}}} \sum_{i=1}^{d} \sum_{\ell=0}^{\nu_{i}-1} k(\alpha) \alpha_{i} \alpha_{j} \frac{m_{\nu}(a)}{\nu !} H_{1}^{\nu_{1}} \ldots H_{i-1}^{\nu_{i-1}} H_{i}^{\ell} T_{i}^{\nu_{i}-\ell-1} T_{i+1}^{\nu_{i+1}} \ldots T_{d}^{\nu_{d}} .
\end{aligned}
$$


ii) $\left[T_{j}, E_{k}(b, Q)\right]=b_{j} E_{k}(b, Q)-S_{k, j}(b, Q)$, where

$S_{k, j}(b, Q)=$

$$
\begin{aligned}
& \sum_{\alpha \in R_{+}} k(\alpha) \alpha_{j} I_{k}(b, Q) \\
& -B_{\alpha} \sum_{\substack{\nu \in \mathbb{N}^{d} \\
\alpha \in R_{+}}} \sum_{i=1}^{d} \sum_{\ell=0}^{\nu_{i}-1} k(\alpha) \alpha_{i} \alpha_{j} \frac{m_{\nu}(b)}{\nu !} Z_{1}^{\nu_{1}} \ldots Z_{i-1}^{\nu_{i-1}} Z_{i}^{\ell} Q_{i}^{\nu_{i}-\ell-1} Q_{i+1}^{\nu_{i+1}} \ldots Q_{d}^{\nu_{d}} .
\end{aligned}
$$

Proof. Using (8) and Lemma 6 i), we obtain

$$
\begin{aligned}
& {\left[E_{k}(a, T), Q_{j}\right]} \\
& =\sum_{\nu \in \mathbb{N}^{d}} \frac{m_{\nu}(a)}{\nu !}\left[T^{\nu}, Q_{j}\right] \\
& =\sum_{\nu \in \mathbb{N}^{d}} \frac{m_{\nu}(a)}{\nu !} \nu_{j} T_{1}^{\nu_{1}} \ldots T_{i-1}^{\nu_{i-1}} T_{i}^{\nu_{i}-1} T_{i+1}^{\nu_{i+1}} \ldots T_{d}^{\nu_{d}} \\
& \quad+B_{\alpha} \sum_{\nu \in \mathbb{N}^{d}} \sum_{i=1}^{d} \sum_{\ell=0}^{\nu_{i}-1} \sum_{\alpha \in R_{+}} k(\alpha) \alpha_{i} \alpha_{j} \frac{m_{\nu}(a)}{\nu !} H_{1}^{\nu_{1}} \ldots H_{i-1}^{\nu_{i-1}} H_{i}^{\ell} T_{i}^{\nu_{i}-\ell-1} T_{i+1}^{\nu_{i+1}} \ldots T_{d}^{\nu_{d}} .
\end{aligned}
$$

Applying the relation

$$
\frac{\partial}{\partial w_{j}} E_{k}(z, w)=z_{j} E_{k}(z, w)-\sum_{\alpha \in R_{+}} k(\alpha) \alpha_{j} I_{k}(z, w) ; \quad z, w \in \mathbb{C}^{d},
$$

we obtain

$$
\left[E_{k}(a, T), Q_{j}\right]=a_{j} E_{k}(a, T)-R_{k, j}(a, T) .
$$

This proves i). Similarly, we can prove ii).

Remark. If $d=1$ and $G=\mathbb{Z}_{2}[\mathbf{2 1}]$, we have

$$
\begin{aligned}
R_{\gamma}\left(a, T_{\gamma}\right) & =\frac{2 \gamma}{2 \gamma+1} a(I-B) \Im_{\gamma+\frac{1}{2}}\left(a T_{\gamma}\right), \\
S_{\gamma}(b, Q) & =\frac{2 \gamma}{2 \gamma+1} b(I-B) \Im_{\gamma+\frac{1}{2}}(b Q),
\end{aligned}
$$

where $B f(x)=f(-x)$.

Since $E_{k}(a, 0)=1$, the Dunkl kernel $E_{k}(a, z) ; a, z \in \mathbb{C}^{d}$, is a unit in the integral domain formal power series over $\mathbb{C}^{d}$. We define

$$
E_{k}^{-1}(a, z):=\sum_{\nu \in \mathbb{N}^{d}} \frac{t_{\nu}(a)}{\nu !} z^{\nu}
$$

Writing

$$
E_{k}(a, z) E_{k}^{-1}(a, z)=E_{k}^{-1}(a, z) E_{k}(a, z)=1,
$$


we obtain

$$
t_{0}(a)=1, \sum_{\nu \in \mathbb{N}^{d}}\left\{\sum_{s \leq \nu}\left(\begin{array}{c}
\nu \\
s
\end{array}\right) m_{\nu-s}(a) t_{s}(a)\right\} \frac{z^{\nu}}{\nu !}=1 .
$$

Thus $\left\{t_{\nu}(a)\right\}_{\nu \in \mathbb{N}^{d}}$ is a sequence of moment functions in $a$ determined by

$$
t_{0}(a)=1, t_{\nu}(a)=-\sum_{s \leq \nu-1}\left(\begin{array}{c}
\nu \\
s
\end{array}\right) m_{\nu-s}(a) t_{s}(a) .
$$

The function $E_{k}^{-1}(a, z)$ occurs in the generalized Weyl commutation relations for the Dunkl kernel.

Theorem 2. Let $a, b \in \mathbb{C}^{d}$, then:

i) $E_{k}(b, Q) E_{k}(a, T)=E_{k}(a, T) E_{k}\left(b, P_{a}\right), \quad P_{a}=\left(P_{a, 1}, \ldots, P_{a, d}\right)$, where

$$
P_{a, j}=Q_{j}-a_{j} I+E_{k}^{-1}(a, T) R_{k, j}(a, T) .
$$

ii) $E_{k}(a, T) E_{k}(b, Q)=E_{k}(b, Q) E_{k}\left(a, L_{b}\right), \quad L_{b}=\left(L_{b, 1}, \ldots, L_{b, d}\right)$, where

$$
L_{b, j}=T_{j}+b_{j} I-E_{k}^{-1}(b, Q) S_{k, j}(b, Q) .
$$

iii) $E_{k}(a, Q) E_{k}(b, Q)=E_{k}(a \# b, Q), E_{k}(a, T) E_{k}(b, T)=E_{k}(a \# b, T)$, where $a \# b$ is the convolution of $a$ and $b$ given by

$$
m_{\nu}(a \# b)=\sum_{s \leq \nu}\left(\begin{array}{c}
\nu \\
s
\end{array}\right) m_{s}(a) m_{\nu-s}(b) .
$$

Proof. We shall prove i), ii) follows in the same way. For $j=1,2, \ldots, d$, we have

$$
E_{k}^{-1}(a, T) Q_{j} E_{k}(a, T)=E_{k}^{-1}(a, T)\left\{E_{k}(a, T) Q_{j}-\left[E_{k}(a, T), Q_{j}\right]\right\} .
$$

Using Lemma 7 i), we obtain

$$
E_{k}^{-1}(a, T) Q_{j} E_{k}(a, T)=Q_{j}-a_{j} I+E_{k}^{-1}(a, T) R_{k, j}(a, T) .
$$

Thus implies that for $\nu \in \mathbb{N}^{d}$ :

$$
E_{k}^{-1}(a, T) Q^{\nu} E_{k}(a, T)=P_{a}^{\nu}, P_{a}=\left(P_{a, 1}, \ldots, P_{a, d}\right),
$$

where

$$
P_{a, j}=Q_{j}-a_{j} I+E_{k}^{-1}(a, T) R_{k, j}(a, T) .
$$

Multiplying by $\frac{m_{\nu}(b)}{\nu !}$ and summing, we get

$$
E_{k}^{-1}(a, T) E_{k}(b, Q) E_{k}(a, T)=E_{k}\left(b, P_{a}\right) .
$$

Then i) follows upon multiplication by $E_{k}(a, T)$.

iii) It suffices to prove the first relation. 
Using (8) and (12), we can write

$$
\begin{aligned}
E_{k}(a, Q) E_{k}(b, Q) & =\sum_{\nu, s \in \mathbb{N}^{d}} \frac{m_{\nu}(a) m_{s}(b)}{\nu ! s !} Q^{\nu+s} \\
& =\sum_{\nu \in \mathbb{N}^{d}}\left\{\sum_{s \leq \nu}\left(\begin{array}{c}
\nu \\
s
\end{array}\right) m_{s}(a) m_{\nu-s}(b)\right\} \frac{Q^{\nu}}{\nu !} \\
& =\sum_{\nu \in \mathbb{N}^{d}} \frac{m_{\nu}(a \# b)}{\nu !} Q^{\nu} .
\end{aligned}
$$

Thus we obtain

$$
E_{k}(a, Q) E_{k}(b, Q)=E_{k}(a \# b, Q)
$$

Remarks. 1) In the classical case $(k=0)$ [13, p. 223], the Weyl commutation relations are given by

$$
\begin{aligned}
& e^{\langle a, P\rangle} e^{\langle b, Q\rangle}=e^{\langle a, b\rangle} e^{\langle b, Q\rangle} e^{\langle a, P\rangle}, \\
& e^{\langle a, P\rangle} e^{\langle b, P\rangle}=e^{\langle a+b, P\rangle} \\
& e^{\langle a, Q\rangle} e^{\langle b, Q\rangle}=e^{\langle a+b, Q\rangle}
\end{aligned}
$$

where $P=\left(\frac{\partial}{\partial x_{1}}, \ldots, \frac{\partial}{\partial x_{d}}\right)$ and $Q=\left(Q_{1}, \ldots, Q_{d}\right)$.

2) If $d=1$ and $G=\mathbb{Z}_{2}[\mathbf{2 1}]$, the Weyl commutation relations are given by

$$
\begin{aligned}
& E_{\gamma}(b Q) E_{\gamma}\left(a T_{\gamma}\right)=E_{\gamma}\left(a T_{\gamma}\right) E_{\gamma}\left(b P_{a}\right) \\
& E_{\gamma}\left(a T_{\gamma}\right) E_{\gamma}(b Q)=E_{\gamma}(b Q) E_{\gamma}\left(a L_{b}\right)
\end{aligned}
$$

where

$$
P_{a}=Q-a I+\frac{2 \gamma}{2 \gamma+1} a E_{\gamma}^{-1}\left(a T_{\gamma}\right)(I-B) \Im_{\alpha+1}\left(a T_{\gamma}\right)
$$

and

$$
L_{b}=T_{\gamma}+b I-\frac{2 \gamma}{2 \gamma+1} b E_{\gamma}^{-1}(b Q)(I-B) \Im_{\alpha+1}(b Q) .
$$

Acknowledgments. It is our pleasure to thank Professor Dr. M. Rösler and Professor M. Voit for their interesting discussions. 


\section{References}

[1] N. Aronszajn, Theory of reproducing kernels, Trans. Amer. Math. Soc., 68 (1950), 337-404, MR 0051437, Zbl 0037.20701.

[2] T.H. Baker and P.J. Forrester, The Calogero-Sutherland model and generalized classical polynomials, Comm. Math. Phys., 188 (1997), 175-216, MR 1471336, Zbl 0903.33010.

[3] _ The Calogero-Sutherland model and polynomials with prescribed symmetry, Nucl. Phys., B 492 (1997), 682-716, MR 1456121, Zbl 0986.33500.

[4] V. Bargmann, On a Hilbert space of analytic functions and an associated integral transform, Comm. Pure Appl. Math., 14 (1961), 187-214, MR 0157250, Zbl 0107.09102.

[5] C.A. Berger and L.A. Coburn, Toeplitz operators on the Segal-Bargmann space, Trans. Amer. Math. Soc., 301 (1987), 813-829, MR 0882716, Zbl 0625.47019.

[6] I. Cherednik, A unification of the Knizhnik-Zamolodchikov equation and Dunkl operators via affine Hecke algebras, Invent. Math., 106 (1991), 411-432, MR 1128220, Zbl 0742.20019.

[7] F.M. Cholewinski, Generalized Fock spaces and associated operators, SIAM, J. Math. Anal., 15 (1984), 177-202, MR 0728694, Zbl 0596.46017.

[8] L.A. Coburn and J. Xia, Toeplitz algebras and Rieffel deformations, Comm. Math. Phys., 168 (1995), 23-38, MR 1324389, Zbl 0834.47021.

[9] C.F. Dunkl, Differential-difference operators associated to reflection groups, Trans. Amer. Math. Soc., 311 (1989), 167-183, MR 0951883, Zbl 0652.33004.

[10] _ Integral kernels with reflection group invariance, Canad. J. Math., 43 (1991), 1213-1227, MR 1145585, Zbl 0827.33010.

[11] P. Etingof and V. Ginzburg, Symplectic reflection algebras, Calogero-Moser space, and deformed Harish-Chandra homomorphism, Invent. Math., 147 (2002), 243-348, MR 1881922.

[12] V. Fock, Verallgemeinerung und Lösung der Diracschen statistischen Gleichung, Z. Phys., 49 (1928), 339-357.

[13] T. Hida, Brownian motion, Applications of Mathematics, 11, Springer-Verlag, New York-Berlin, 1980, MR 0562914, Zbl 0432.60002.

[14] L. Lapointe and L. Vinet, Exact operator solution of the Calogero-Sutherland model, Comm. Math. Phys., 178 (1996), 425-452, MR 1389912, Zbl 0859.35103.

[15] N. Obata, White noise calculus and Fock space, Lecture Notes in Mathematics, 1577, Springer-Verlag, Berlin, 1994, MR 1301775, Zbl 0814.60058.

[16] E.M. Opdam, Harmonic analysis for certain representations of graded Hecke Algebras, Acta Math., 175 (1995), 75-121, MR 1353018, Zbl 0836.43017.

[17] M. Rösler, Generalized Hermite polynomials and the heat equation for Dunkl operators, Comm. Math. Phys., 192 (1998), 519-542, MR 1620515, Zbl 0908.33005.

[18] _ Positivity of Dunkl's intertwining operator, Duke Math. J., 98 (1999), 445463, MR 1695797, Zbl 0947.33013.

[19] M. Rösler and M. Voit, Markov processes related with Dunkl operators, Adv. Appl. Math., 21 (1998), 575-643, MR 1652182, Zbl 0919.60072.

[20] Biorthogonal polynomials associated with reflection groups and a formula of Macdonald, J. Comp. Appl. Math., 99 (1998), 337-351, MR 1662706, Zbl 0928.33012. 
[21] M. Sifi and F. Soltani, Generalized Fock spaces and Weyl relations for the Dunkl kernel on the real line, J. Math. Anal. Appl., 270 (2002), 92-106, MR 1911753, Zbl 1012.46033.

Received July 25, 2002 and revised June 26, 2003.

Department of Mathematics

FACUlty OF SCIENCES OF TUNis

UNIVERSITY OF TUNIS-EL MANAR

1060 TUNIS

TUNISIA

E-mail address: Fethi.Soltani@fst.rnu.tn 